\title{
Arduino Based LPG gas Monitoring \& Automatic Cylinder booking with Alert System
}

\author{
R.Naresh Naik ${ }^{1}$, P.Siva Nagendra Reddy ${ }^{2}$,S.Nanda Kishore ${ }^{3}$, \\ K.Tharun Kumar Reddy ${ }^{4}$ \\ 1,2,4 (Assistant Professor, Department of ECE, Kuppam Engineering College, Kuppam,Chittoor,A.P,India) \\ ${ }_{3}^{3}$ (Associate Professor, Department of ECE, Kuppam Engineering College, Kuppam,Chittoor,A.P,India)
}

\begin{abstract}
There is a rapid development in technology which influencing the human life in several aspects due to rapid development in different fields but we still need to adopt that technology such that we can make human life more easier to live. In our Country it is not possible to supply LPG through Pipes to each and every home as production of LPG is too short. At present we are having an system Advance LPG cylinder booking through IVRS or online which is most difficult for the illiterate and busy schedule people to book the LPG cylinder in advance. Another Major problem LPG cylinder users facing is "They don't know exactly the status of LPG gas completion" makes even more delay in booking the cylinder which is uncomfortable most of the times. Now a days we are having a IVRS system in which customer needs to go through few steps in accordance with the Automatic voice which also includes selecting options. Most of the illiterate people can't even complete the booking due to this reason and also most of the times these landline phones are either busy due to congested calls or phones not working due to some technical issues. This paper proposes a system that will make entire $L P G$ cylinder booking procedure automated without human intervention. This system continuously measures the weight of the cylinder and once it reaches minimum threshold it will automatically sends message to the authorized LPG Agent so that they can deliver the LPG cylinder in time. Along with the Automated cylinder booking we also designed feature related to the safety of the user in which it continuously monitor the leakage of LPG gas and alerts the user regarding leakage to avoid major accidents which costs human lives mostly.
\end{abstract}

Keywords: Arduino Mega, Temperature sensor, GSM Modem, Gas Sensor, Load Cell.

\section{Introduction}

There are approximately 30crore LPG users in the country in which mostly $40 \%$ of the population. The Several standards have been implemented for the gas leakage detection system. The existing systems provides an alarm system which is mainly meant to detect an Gas leakage in the house and commercial premises

The objective of the proposed system is to continuously measure the weight of the cylinder and as soon as it reaches the minimum threshold it will automatically sends an SMS alert to the user as well as Authorized LPG agent so that they can act accordingly. This system also designed to detect LPG gases such as propane and butane. The allowed level for butane is 600ppm above which it is considered to be of high level and poses a danger. The threshold level of weight of the cylinder is used for automatic cylinder booking. The main aim of this project is to monitor for liquid petroleum gas (LPG) leakage to avoid major fire accidents and also facilitating safety precautions where security has been an important issue and automatic cylinder booking without human intervention. The system detects the leakage of the LPG using gas sensor and alerts the consumer about the gas leakage by sending SMS. The system measure the weight of cylinder by using weight sensor and display corresponding weight in LPG display. The proposed system uses the GSM Modem to alert the person about the gas leakage via SMS and status of automatic cylinder booking.

When the system identifies that LPG concentration in the air reaches the specified level then it alert the consumer by sending SMS to registered mobile phone and alert the people at home by activating the alarm which includes Buzzer simultaneously and also display the same message on LCD to take the necessary action and switch on the exhaust fan or opening windows to decrease the gas concentration in the air.

\section{Literature Survey}

In the year 2011, A. MAHALINGAM, R. T. NAAYAGI,1, N. E. MASTORAKIS, "Design and Implementation of an Economic Gas Leakage Detector", This project developed system to detect the gas leakage and providing immediate alarm or intimation to the user.

Later in 2013, few people developed the design proposed for home safety. This system detects the leakage of the LPG and alerts the consumer about the leak by buzzer. This project was developed using microcontroller ARM version 7 processor and simulated using Keil software. 
In the year 2014, Hitendra Rawat, Ashish Kushwah, Khyati Asthana, Akanksha Shivhare, designed a system, They provided security issues against thieves, leakage and fire accidents. In those cases their system sends SMS to the emergency number provided to it.

In the proposed system we have designed "LPG gas monitoring and automatic cylinder booking with alert system". These report focus on detection of economic fuels like petroleum, liquid petroleum gas, alcohol..etc., and alert the surrounding people about the leakage through SMS. It also sense surrounding temperature, so that no fire accidents occurs.

The one more important feature is automatic cylinder booking by noticing the current expenditure of LPG gas in our daily life. These projects alert the user by sending message to mobile through SMS in three conditions.

They are

- When LPG gas weight reaches to maximum threshold value.

- When the LPG gas exceed its peak value.

- When the temperature exceed more than room temperature.

These project gives alert message by buzzing the buzzer and trough SMS to the house holders. We also provide automatic doors and windows opening, so that the compressed gas can spread in to air freely. Hence a fire accident does not occurs.

\section{Design And Implementation}

This proposed method consists of gas leakage detection system, weight measurement module, microcontroller, GSM module and alert system.

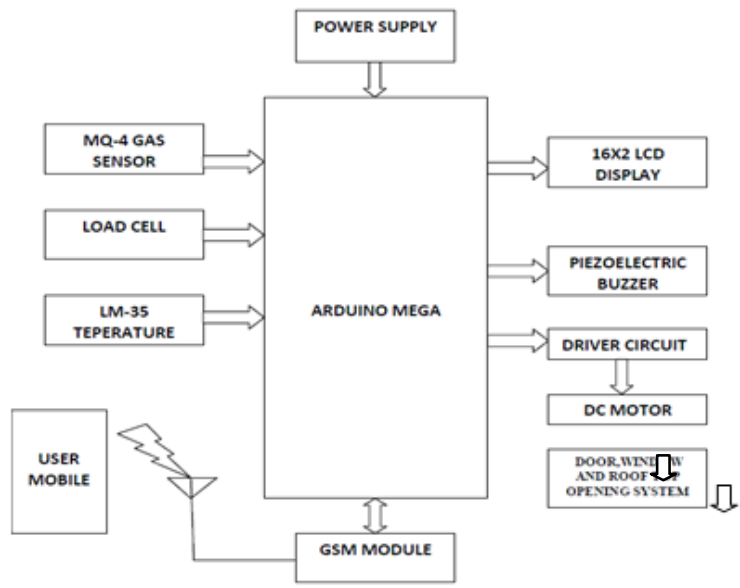

Fig 1: Block Diagram of Proposed System

The main basic Arduino Mega2560 micro controller requires the power supply ranging from 7-12 volts which can be build by using different components like step down transformer, rectifier, filter and regulator which are readily available as adapters these days. Supply can be either from an ac to dc adapter or battery. The board can operate on at $7-12$ volts. If voltage $<7 \mathrm{~V}$ then board becomes unstable. If voltage $>12 \mathrm{~V}$ then board get damaged.

The Main platform we are using to build the project is Arduino Mega 2560 which provides us the flexibility to write the code effectively in convenient way and also it will provides us features like Inexpensive, Cross platform, Simpler and clear programming environment, Open source and extensible software, Easy for beginners, The Arduino Mega 2560 is a microcontroller board based on the ATmega2560 (datasheet).It contains everything needed to support the microcontroller; simply connect it to a computer with a USB cable or power it with a AC to-DC adapter or battery to get started. With the above features it force us to use in our project design.

The other main component we are using in our project is use of Load cell. A load cell is a transducer that is used to convert a force into electrical signal, which is used to measure of a LPG gas cylinder weight so that we can expect and alert the user with in how many days the cylinder is about to empty. There are different Load cells available in the market with different weight measurement capabilities.

The Gas Sensor is also one of the components used to detect the leakage of the LPG Gas (Methane \& Propane) which converts one form of the signal into other form. There are different type of sensors available in the market we make use of few of them in our project such as Methane (MQ4) Sensor and Temperature Sensor (LM35).The MQ4 is used in gas leakage detecting equipments are suitable for detecting of CH4,Natural gas and 
to avoid the noise of alcohol and cooking fumes and cigarette smoke. The MQ-4 can detect natural gas concentrations from 200 to 10000ppm. High sensitivity to CH4, Natural gas. MQ 4 has Fast response, Stable and long life. The LM35 series is a precision IC temperature devices with an output voltage linearlyproportional to the Centigrade temperature.

LCD (Liquid Crystal Display) is used to show the output of the results of Different sensor values and various results to show of size about 32 ASCII character in 2 lines commonly used one is 16x2 LCD modules.

We are using GSM Modem to alert the user by sending SMS(Short Message Service) about Gas Leakage and LPG Gas Completion Status.GSM (Global System for Mobile communication) is a digital mobile telephony system that is widely used in England and different parts of the world. GSM uses of time division multiple access (TDMA) and is the most widely used of the technologies (TDMA, GSM, and CDMA). GSM digitizes and syncs data, then sends it to a channel with two other streams of user data, each in its own time slot. It operates at either the $800 \mathrm{MHz}$ or $1800 \mathrm{MHz}$ frequency band.

\section{Methane gas sensor:}

MQ-4 is a Sensor for Natural Gases Sensitive material. MQ-4 gas sensor is $\mathrm{SnO}$, which has lower conductivity in clear air. When the target combined gas exist, the sensor's conductivity is heavier with the gas concentration rising. we used simple circuit to convert respective output signal according to concentration level. MQ-4 gas sensor has high sensitive to Methane, Propane and Butane. The sensor can be used to detect different combustible gas, especially Methane; it is with cost effective and useful for so many applications.

\section{LM35 Temperature sensor}

LM-35 is a integrated circuit sensor that can be used to measure the temperature with an electrical output proportional to the temperature (in centigrade's).LM35 generates a higher output voltage than thermocouples and may not require that the output voltage be amplified.

Its output voltage is proportional to Celsius temperature, Scale factor is $.01 \mathrm{v} /$ centigrade It consumes approximately 60 micro amps from its supply which is very low and possesses a low self-heating capability.

\section{LCD Display}

LCD stands for Liquid Crystal Display.. They have become very common with industry by clearly replacing the use of Cathode Ray Tubes (CRT). CRT consumes more power than LCD and also bigger and heavier. We all know about LCD's, but no one knows the exact working of it. LCD is finding wide spread use replacing LEDs (seven segments or other multi segment LEDs) due to the following reasons:

- The declining prices of LCDs.

- The ability to display numbers, characters and graphics related data. This is in contrast to LEDs, which are limited to numbers and a few characters.

- Incorporation of a controller into the LCD, thereby making the CPU to keep displaying the data.

- Ease to program for characters, strings and graphics related data.

These are specialized for being used with the microcontrollers, which makes that they cannot be activated by standard IC circuits. They are used for writing different messages on a miniature LCD.

\section{Load cell}

As per dictionary, a load cell is described as a "weight measurement device necessary for electronic scales that display weights in digits." However, load cell is not restricted to weight measurement in electronic scales.

Load cell is a passive transducer or sensor which converts applied force into electrical signals. They are also referred to as "Load transducers".

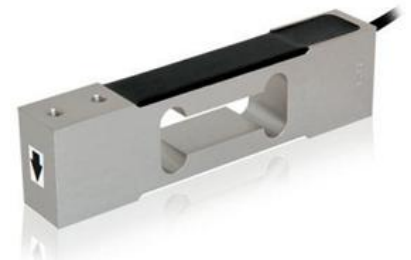

Fig 2: Load Cell

Load cells use different operating principles, viz.,

- Load Cells based on fluid pressure

- Load Cells based on elasticity

- Load Cells based on magnetostriction effect or piezoelectric effect 
However, the only load cells which are prevalent are the load cells based on strain gages. Hence, the term 'load cell' means 'strain gage-based load cells'. The reason behind the wide adoption of strain gage-based load cells is their characteristics

- Highly precise and linear measurements

- Little influence due to temperature changes.

- Small size compared with other types of load cells.

- Long operating life due to lack of moving parts or any parts that generate friction.

- Ease in production due to small number of components.

- Excellent fatigue characteristics

Strain-gauge load cells convert the load acting on them into electrical signals. The measuring is done with very small resistor patterns called strain gauges - effectively small, flexible circuit boards. The gauges are bonded onto a beam or structural member that deforms when weight is applied, in turn deforming the straingauge. As the strain gauge is deformed, it's electrical resistance changes in proportion to the load. The change to the circuit caused by force is much smaller than the changes caused by variation in temperature. Higher quality load cells cancel out the effects of temperature using two techniques. By matching the expansion rate of the strain gauge to the expansion rate of the metal it's mounted on, undue strain on the gauges can be avoided as the load cell warms up and cools down. The most important method of temperature compensation involves using multiple strain gauges, which all respond to the change in temperature with the same change in resistance. Some load cell designs use gauges which are never subjected to any force, but only serve to counterbalance the temperature effects on the gauges that measuring force. Most designs use 4 strain gauges, some in compression, and some under tension, which maximizes the sensitivity of the load cell, and automatically cancels the effect of temperature. It is often easy to measure the parameters like length, displacement, weight etc that can be felt easily by some senses. However, it is very difficult to measure the dimensions like force, stress and strain that cannot be really sensed directly by any instrument. For such cases special devices called strain gauges are very useful. There are some materials whose resistance changes when strain is applied to them or when they are stretched and this change in resistance can be measured easily. For applying the strain you need force, thus the change in resistance of the material can be calibrated to measure the applied force. Thus the devices whose resistance changes due to applied strain or applied force are called as the strain gauges.

\section{Piezoelectric buzzer}

Buzzer is an audio signaling device. The typical uses of buzzers are for alarms, timers and confirmation of user input such as a mouse click or keystroke. The project used an electronic type of buzzer which is a piezoelectric element that driven by an micro-controller signals. Peizo buzzer is based on the inverse principle of peizo electricity discover in 1880 by Jacques and Pierre Curie. It is the phenomenon of generating electricity when mechanical pressure is applied to the certain materials and the vice versa. such materials called Piezoelectric materials. When an alternating electric field subjected to the material they stretch or compress accordance with the frequency of the signal thereby producing sound.

\section{Arduino MEGA}

In this proposed system Arduino Mega is used as a controller. Arduino is an well equipped Open-Source Prototype Platform based on easy-to-use hardware and software. Arduino boards are able to read inputs - light sensor, or a Twitter message - and turn it into an output - activating a motor, publishing anything online.

The Arduino software is easy-to-use for beginners. It will simplify the process of creating a control environment by providing the standard and flexible board that can be reusable programmed and connected to the system without any necessity of PCB design. Inexpensive, Cross-platform, Simple programming environment, Open source extensible software, Open source and extensible hardware. It is flexible enough for advanced users. It works on platforms like Mac, Windows and Linux. Arduino is a key tool to learn new things.

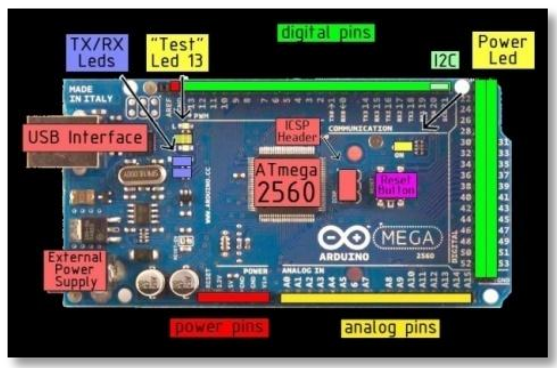

Fig 3: Arduino Mega2560 Board 


\section{GSM Module}

GSM (Global System for Mobile) / GPRS (General Packet Radio Service) TTL modem s SIM900 quad-band GSM / GPRS device, works on frequencies $850 \mathrm{MHZ}, 900 \mathrm{HZ}, 800 \mathrm{MHZ}$ and $1900 \mathrm{HZ}$. It is very compact in size and easy to use as plug in GSM Modem. The Modem is designed with 3V3 and 5V DC TTL interfacing circuitry, which allows User to directly interface with $5 \mathrm{~V}$ microcontrollers (PIC, AVR, Arduino, 8051, etc.) as well as 3V3 Microcontrollers (ARM, ARM Cortex XX, etc.).

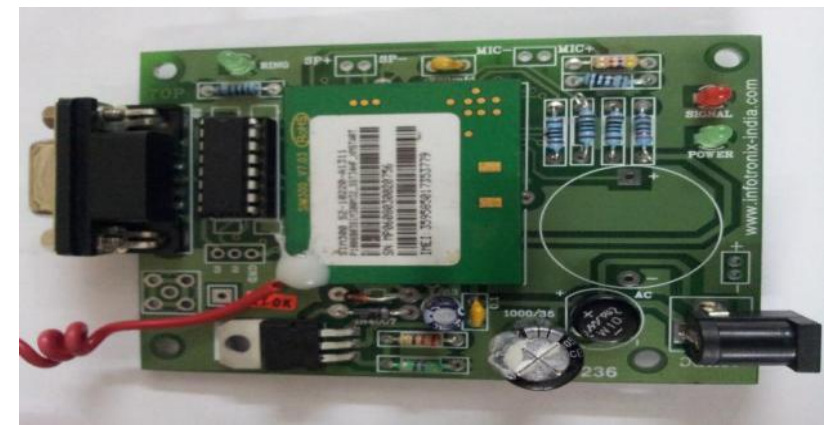

Fig 4: GSM SIM800 Module

The baud rate can be configurable from 9600-115200 bps through AT (Attention) commands. This GSM/GPRS TTL Modem has internal TCP/IP stack to enable User to connect with internet through GPRS feature. It is suitable for SMS as well as DATA transfer application in mobile phone to mobile phone interface. The modem can be interfaced with a Microcontroller using USART (Universal Synchronous Asynchronous Receiver and Transmitter) feature.

\section{Results \& Discussions}

In Arduino Based LPG gas Monitoring \& Automatic Cylinder booking with Alert System MQ-4 gas sensor,LM-35 Temperature sensor,10 kg load cell( for prototype) as input devices and Piezoelectric buzzer, 16x2 LCD display and GSM module used as output devices.

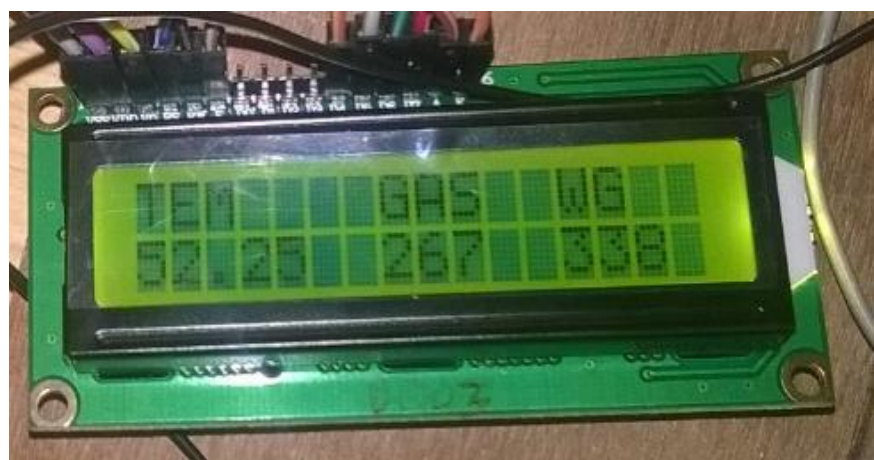

Fig 5: LCD showing output of Temperature, gas level and weight

Actually the room temperature is $25^{\circ} \mathrm{C}$, but we increased temperature of LM35 upto $53^{\circ} \mathrm{c}$. Initially before gas leakage the output of gas sensor is $0 \mathrm{ppm}$, but when it sense gas the output is $267 \mathrm{ppm}$. We have placed $10 \mathrm{~kg}$ load cell, so the maximum capacity is $10 \mathrm{~kg}$ load. 338 is in the units of grams of weight.

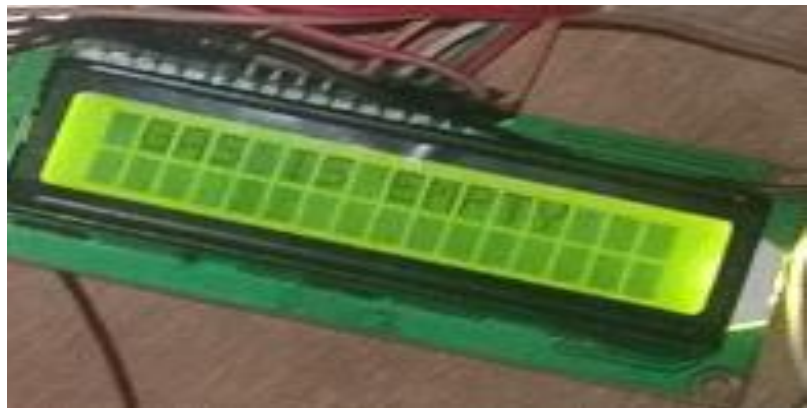

Fig 6: Output showing Status of LPG cylinder 
The above figure represents SMS messages in user mobile phone which is send by GSM module for different kinds of input reaction in our project. The message "GAS IS LOW RECHARGE SOON" is sent to the user when the LPG gas reaches to minimum threshold level. So the user comes to know to when to book the cylinder to avoid delay in delivering cylinder. The message "GAS IS EMPTY RECHARGE IMMEDIETLY" is sent when the weight of the gas is fully empty.

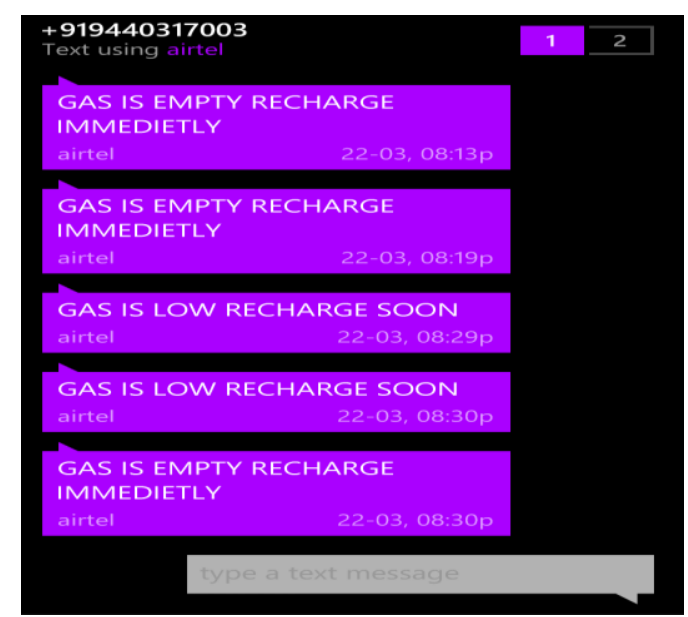

Fig 7: SMS's sent to the user mobile

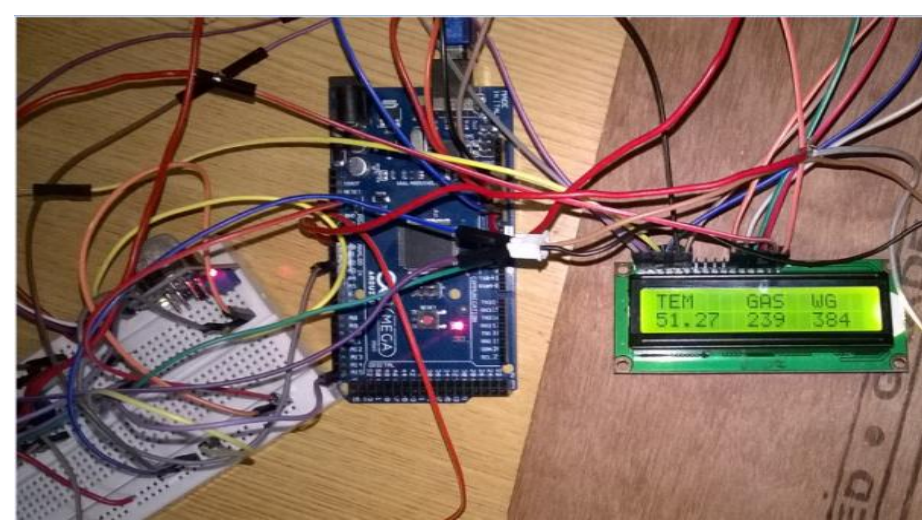

Fig 8: Initial Design of the Project

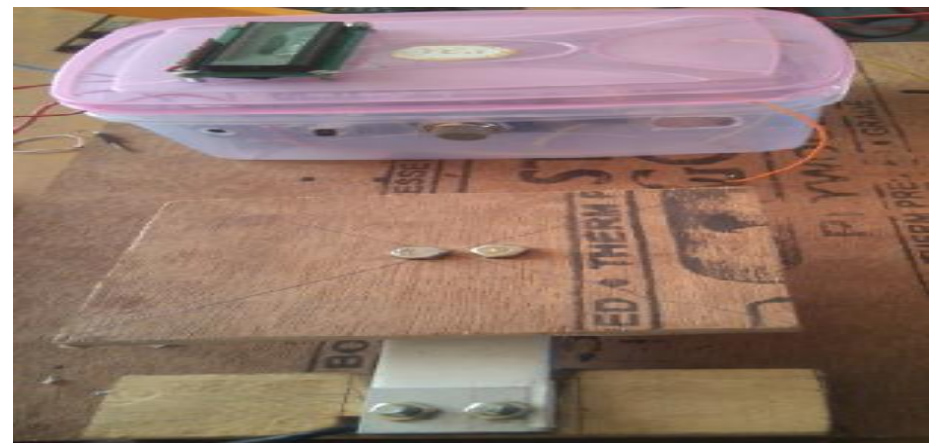

Fig 9: Final Design of the Project

\section{Conclusion}

As we shorted out the problems faced by LPG gas consumers so we come up with some solutions to meet the few requirements of them, as we made our system is completely automate the process of refill booking without human intervention. Our system is also reasoned to help customers to upgrade their safety norms, act in accordingly with minimum requirements on environmental issues and mostly the basic function being prevented by major disasters and protect life and property from reputed Accidents. The primary objective of our project is to measure the gas present in the cylinder when weight of the cylinder is below the fixed load, this can be done using the weight sensors. The gas retailer gets the order for a new cylinder and the house owner (consumer) receives the message regarding the status and the secondary objective is to provide any malfunction 
in gas servicing system in order to prevent damage or explosion of LPG. Thus the system developed by us will somehow help the LPG Gas Consumers to lead a comfortable life.

\section{Future Scope}

This monitoring system can be further enhanced by using Bluetooth in place of GSM to send the alert messages to user, which supports the another real-time application. For industrial purposes mobile robot can be developed for detecting multiple gas concentrations. Addition of load cell can also be used as pressure sensor which detects the amount of gas in the cylinder and also detects high pressure gas in cylinder pipe, displaying the alert messages via SMS and LCD displays.

\section{Acknowledgements}

We thank to Dr.G.N.Kodanda Ramaiah, Professor \& HOD, and our colleagues from Department of ECE, Kuppam Engineering College who provided insight and expertise that greatly assisted the research, although they may not agree with all of the interpretations/conclusions of this paper.

\section{References}

[1]. K. Galatsis, W. Woldarsla, Y.X. Li and K. Kalantar-zadeh, "A Vehicle air quality monitor using gas sensors for improved safety", report in Recent Researches in Applications of Electrical and Computer Engineering.

[2]. K. Galatsis, W. Wlodarsla, K. Kalantar-Zadeh and A. Trinchi, "Investigation of gas sensors for vehicle cabin air quality monitoring", National Conference on Synergetic Trends in engineering and Technology (STET-2014), International Journal of Engineering and Technical Research ISSN: 2321-0869

[3]. "Smart Gas Cylinder Using Embedded System", Issn (Online) 2321 - 2004 Issn (Print) 2321 - 5526, International Journal Of Innovative Research In Electrical, Electronics, Instrumentation And Control Engineering Vol. 2, Issue 2, February 2014.

[4]. "Design and Implementation of an Economic Gas Leakage Detector" A. MAHALINGAM, R. T. NAAYAGI,1, N. E. MASTORAKIS§ Department of Engineering Systemsschool of Engineering, University of Greenwich (Medway Campus)Chatham Maritime, Kent ME4 4TBUNITED KINGDOM, article in Recent Researches in Applications of Electrical and Computer Engineering.

[5]. Fraiwan, L.; Lweesy, K.; Bani-Salma, A.; Mani, N, “A wireless home safety gas leakage detection system”, Proc. of 1st Middle East Conference on Biomedical Engineering, pp. 11-14, 2011.

[6]. Johansson, A.; Birk, W.; Medvedev, A., "Model-based gas leakage detection and isolation in a pressurized system via Laguerre spectrum analysis", Proc. of IEEE International Conference on Control Applications, pp. 212-216, 1998.

[7]. Lopes dos Santos, P.; Azevedo-Perdicoúlis, T.- P.; Ramos, J.A.; Jank, G.; Martins de Carvalho, J.L.; Milhinhos, J., "Gas pipelines LPV modeling and identification for leakage detection", Proc. of American Control Conference, pp. 1211-1216, 2010.

[8]. Lopes dos Santos, P.; Azevedo-Perdicoúlis,T,P.Ramos,J.A.; Martins de Carvalho, J.L.; Jank, G.; Milhinhos, "An LPV modeling and identification approach to leakage detection in high pressure natural gas transportation networks", IEEE Transactions on Control Systems Technology, vol. 19, pp. 77-92, 2011.

About Authors:

Mr. R.Naresh Naik received M.Tech in VLSI System Design from JNTUA university. He is working in

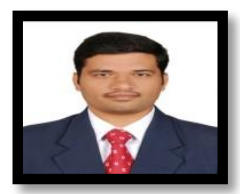

Kuppam Engineering College as assistant Professor in Electronics and Communication Engineering Department. Email-id: nareshkec2015@gmail.com

Mr. Siva Nagendra Reddy received M.Tech in VLSI System Design from JNTUA university. He is working

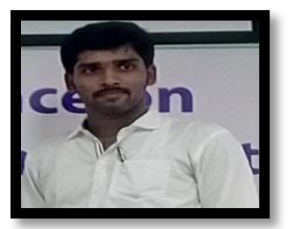
in Kuppam Engineering College as assistant Professor in Electronics and Communication Engineering Department. Email-id: Snreddy715@gmail.com

Mr. S.Nanda Kishore is working in Kuppam Engineering College as associate Professor in Electronics and

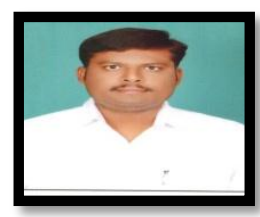

Communication Engineering Department. Email-id: nankishor@gmail.com

Mr. K.Tharun Kumar Reddy received M.Tech in Embedded System from JNTUA university. He is working

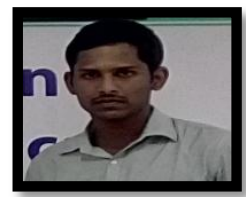
in Kuppam Engineering College as assistant Professor in Electronics and Communication Engineering Department. Email-id: kethireddy.tharun@gmail.com 